\title{
Les traces de Pedro LEMEBEL
}

Entretien avec Fernanda CARVAJAL

Fernanda Carvajal

\section{(2) OpenEdition}

Journals

Édition électronique

URL : http://journals.openedition.org/conflits/19500

DOI : $10.4000 /$ conflits. 19500

ISSN : $1777-5345$

Éditeur :

CCLS - Centre d'études sur les conflits lilberté et sécurité, L'Harmattan

Édition imprimée

Date de publication : 15 juillet 2017

Pagination : 191-204

ISBN : 978-2-343-12713-2

ISSN : $1157-996 \mathrm{X}$

Référence électronique

Fernanda Carvajal, « Les traces de Pedro LEMEBEL », Cultures \& Conflits [En ligne], 105-106 printemps/été 2017, mis en ligne le 15 juillet 2017, consulté le 30 mars 2021. URL : http:// journals.openedition.org/conflits/19500; DOI : https://doi.org/10.4000/conflits.19500 


\section{Imprésentable LEMEBEL}

Pedro Lemebel (1956-2015) est un artiste et écrivain chilien. D'abord connu pour ses performances menées dans les années 1980, il est l'auteur de plusieurs recueils de chroniques et d'un roman que le public français a pu découvrir dans les années 2000 1. Avant d'être réunies dans des livres, ces chroniques étaient destinées à des émissions de radio et publiées dans des journaux 2. La sexualité, l'bomosexualité, sont des éléments importants dans son ceuvre. Tout comme le regard porté sur diverses formes d'exclusion, de mise au ban. Figure inclassable de la vie culturelle chilienne, Lemebel est aussi, à lui tout seul, une langue. Une langue incisive dont la matière première semble être ce " côté obscur du cour " que d'autres préféreraient passer sous silence. Lemebel écrit et dit ses textes. Il prend la parole et se mêle de tout. De politique aussi. Méprisé par certains, encensé par d'autres, il devient au fil des ans une figure populaire. Ce dossier offre au lecteur français, non familier de son cuvre, des éléments d'introduction, sorte de présentation du plus imprésentable ${ }^{2}$ des artistes chiliens ${ }^{3}$.

1. Lemebel P., Je tremble, ô matador, Paris, 10/18, 2007. Voir également en français la thèse de doctorat : López García I., La question du genre dans les chroniques de Pedro Lemebel, Paris, Université Paris IV - Sorbonne École Doctorale IV, 2007.

2. D’abord publiées au Chili, les œuvres de Lemebel ont fait l'objet de rééditions notamment en Argentine et en Espagne. Parmi d'autres titres, on peut citer : La esquina es mi corazón : crónica urbana, Santiago, Cuarto Propio, 1995 ; Loco afán: crónicas de sidario, Santiago, LOM, 1996 ; De perlas y cicatrices, Santiago, 1998.

3. Dossier préparé par Antonia Garcia Castro et Fernanda Carvajal. 


\title{
Les traces de Pedro LEMEBEL
}

\author{
Entretien avec Fernanda CARVAJAL
}

Fernanda Carvajal (Chili, 1982) est sociologue. Titulaire d'un master en communication et culture, et docteur en sciences sociales. Elle réside depuis 2008 à Buenos Aires. Ses travaux portent sur les liens entre art, politique et sexualité en Amérique $d u$ Sud, à partir des années 1970. Membre du résean de chercheurs Red Conceptualismos del Sur ${ }^{4}$, elle a participé à l'exposition "Perdre la forme bumaine " présentée au Musée Reina Sofía de Madrid, en 2012 (puis à Lima, en 2013, et à Buenos Aires, en 2014). Elle travaille actuellement avec Alejandro de la Fuente à la constitution des archives des interventions faites par Pedro Lemebel dans le cadre du duo conformé avec Francisco Casas ("Yeguas del Apocalipsis "), dans les années 1980, et avec lequel il a fait irruption dans la vie publique chilienne 5. Fernanda Carvajal a connu Pedro Lemebel en 2010. Selon ses mots «ce lien, qui a d'abord été un lien professionnel puis d'amitié, a laissé une marque à la fois sur le plan affectif et dans mon écriture, car on ne pouvait pas rencontrer Lemebel et en sortir indemne ». Cet entretien tient compte de ces liens.

$* * *$

CEC : Pedro Lemebel a publié en France un roman. Cependant, an Chili, il est principalement connu pour ses chroniques et ses performances. Comment définirais-tu Lemebel ? Sa personne? Son ceuvre?

Fernanda Carvajal (F.C.) : Avant toute chose, je dirais que Pedro Lemebel est une sorte d'animal improbable dans une société aussi inégalitaire que la société chilienne. Une société marquée par les préjugés de classe et moralement conservatrice. C'est un point crucial. Les frontières symboliques, matérielles, sexuelles, politiques, qui marquent sa trajectoire, le rendent seulement comparable - peut-être - à Violeta Parra ${ }^{6}$. Bien entendu, il faudrait pou-

4. Il s'agit d'une plateforme internationale, espace de recherche, de débats et de prises de position sur des questions liées à l'art, à la politique, à la politique de l'art, à la manière dont l'art fait de la politique. Voir : https://redcsur.net/

5. On trouvera des informations complémentaires et des images des interventions sur le site en cours d'actualisation : http://www.yeguasdelapocalipsis.cl/

6. Violeta Parra (1917-1967), artiste, musicienne, compositeur, poète chilienne. 
voir étayer cette comparaison, mais disons : chacun, à son époque, à partir d'une position subalterne, en termes de classe et de genre, habite, réinvente et oblige les autres à porter un regard incisif sur le monde d'où il provient, monde populaire, socialement et économiquement violenté. Tous deux s'inscrivent à gauche, tous deux sont proches du Parti communiste, ce qui n'empêche pas les tensions, tous deux accèdent avec insolence à des instances de validation des centres métropolitains, sans perdre leur lucidité, leur méfiance, et surtout, tous deux produisent une œuvre qui engendre des frictions inappropriées et électrisantes entre des éléments de la culture populaire, de la culture de masse, de la haute culture. Et c'est aussi ce qui fait que leurs œuvres figurent parmi les plus singulières et les plus audacieuses de la culture chilienne du XX⿳亠丷厂 ${ }^{\mathrm{e}}$ siècle.

Pedro Lemebel est né en 1952, dans un bidonville de Santiago 7, au sein d'une famille de partisans d'Allende. Comme il l'a souvent signalé dans ses entretiens, sa littérature ne prétendait pas être érudite. En effet, le moins qu'on puisse dire c'est qu'il ne suit aucune voie royale, pas plus qu'il ne circule dans les espaces légitimés de la culture lettrée. Il y va par les bords, en faisant des zigzags. Lemebel est, en effet, le premier de sa famille à aller à l'université. Dans les années 1970, il fait des études de pédagogie appliquée à l'art. Ensuite, pendant la dictature, il travaille pendant un temps comme professeur d'histoire de l'art dans des lycées. Son contact avec la littérature ne survient que plus tard, lorsque le fait d'être un enseignant visiblement homosexuel, dans des écoles sous tutelle pinochetiste, l'oblige à quitter l'enseignement. C'est alors qu'il commence à gagner sa vie comme vendeur ambulant de livres d'occasion, de cartes postales, d'objets artisanaux. C'est à cette période qu'il rejoint le «Coordinador Cultural », un espace fréquenté par des artistes dissidents, issus de divers courants de gauche, mais principalement du Parti communiste et du Mouvement de gauche révolutionnaire (MIR). Il rencontre des écrivains de la SECH (Sociedad Chilena de Escritores), dont la poète et écrivain féministe Carmen Berenguer, avec laquelle il aura une forte complicité littéraire, artistique et politique. En parallèle, il assiste à l'atelier littéraire de l'écrivain Pía Barros, et c'est là qu'il commence à publier. Ses premières nouvelles paraissent dans l'anthologie Incontables de $1986^{8}$. Cette même année, il écrit son célèbre Manifeste, ou Hablo por mi diferencia 9 . Puis, en 1987, il crée avec Francisco Casas, le duo Yeguas del Apocalipsis [Juments de l'Apocalypse]. Ensemble, ils mènent une série d'actions politico-artistiques, ce qui ne l'empêche pas de travailler également seul. Un type d'intervention qu'il maintiendra jusqu'à la fin de sa vie. Ce n'est qu'à partir de 1990 qu'il

7. Ce quartier est le Zanjón de la Aguada, le nom désigne à la fois un cours d'eau et le quartier aux alentours. Synonyme de misère, c'est aujourd'hui aussi le titre d'un recueil de chroniques de Pedro Lemebel.

8. Barros P. (dir.)., Incontables, Santiago, Ergo Sum, 1986.

9. Ce texte «Manifeste, je parle par ma différence» est effectivement célèbre au Chili. Voir des extraits en encadré. Dorénavant : Manifeste. 
commence son travail comme collaborateur de la revue Página Abierta, où il publie ses premières chroniques et - pour la première fois - son Manifeste. Son premier livre, La esquina es mi corazón, paraît en 1995, et marque le début d'une production littéraire prolifique, qui ne s'est pas limitée au format livre, mais qui s'est toujours donnée d'autres supports tels que la presse ou l'émission de radio, qui a mis en valeur la dimension orale de son écriture.

«On serait tombé dans une obscurité de toile d'araignée sur un garçon qui passait par là. On aurait su qu'on était deux étrangers dans une ville où nous sommes tous étrangers, à cette heure où le rideau tombe en deuil. II est minuit à Santiago. Et chaque rue, chaque recoin, chaque ombre, semble être un animal recroquevillé à l'affût. Cette ville est une faune, tellement dangereuse, le souffle des rues a des échos de braquage et des lueurs de lame. Surtout les week-ends, surtout I'hiver, quand sur l'asphalte mouillé les pas résonnent comme des fuites accélérées. Quelqu'un vient, quelqu'un te suit, quelqu'un s'approche avec un désir malandrin et les pires intentions. Et quand le gars te demande une clope, on sait que la flamme de I'allumette va dévoiler un couteau ».

«On le sait, on n'aurait jamais dû s'arrêter. Mais il était tout près, à quelques pas seulement, et j'ai beau lui dire que je fume des Life, histoire qu'il se fasse une idée de ma situation financière, il accepte quand même, il tape la discute, et soudain, il se tait. Il m'écoute en me fixant des yeux. Et moi, je bégaye, je baratine sans faire de pause pour le distraire, en pensant que tout de suite après, $c^{\prime}$ est l'attaque, le coup, la blessure, le sang. Et comme atteint d'une hémorragie verbale, je n'arrête plus de parler, tout en lorgnant du coin de I'œil une issue pour me sauver. Mais le garçon, un tout jeune homme aux yeux de moustique, $\mathrm{m}^{\prime}$ arrête et $\mathrm{m}$ 'assène un : mais je te connais ! ».

«Tu causes à la radio, non ? Ben oui, je dis, en respirant profondément, un peu plus calme. T'avais la frousse? me demande-t-il. Un peu, je réponds. II est vraiment tard, on peut jamais savoir. T'avais pas tort, dit-il en lâchant un rire tout jeune qui illumine de perles la panique du moment. J'allais te clouer là, mon gars, ajoute-t-il en riant et en me montrant une lame en acier qui me fige l'âme. J'allais te zigouiller, mais quand je t'ai entendu parler, je me suis rappelé la radio, et j'ai pigé que c'était la même voix, celle qu'on écoutait en Sibérie. Mais Radio Tierra c'est une onde courte, on pouvait pas l'entendre de si loin. T'as vécu à l'étranger? Mais non, tu parles, je te dis la Sibérie, en prison quoi ! En taule, trois ans, suis sorti y a peu de temps».

Extraits de : Lemebel P. , Solos en la madrugada (o "el pequeño delincuente que soñaba ser feliz") », De perlas y cicatrices, Santiago, LOM, 1996, pp. 147-148.

Il y a peu de temps, dans un entretien réalisé pour les archives «Yeguas del Apocalipsis », la critique littéraire Raquel Olea faisait une remarque intéressante. Elle disait qu'avec le roman Tengo miedo torero, Pedro Lemebel a fait un geste : il s'est inscrit dans l'écriture homosexuelle de la tradition littéraire chilienne, au sein de laquelle, le référent le plus proche est El lugar sin límites (1966) de José Donoso ${ }^{10}$. Et ce d'une manière particulière : en faisant sortir « la folle », la figure de la folle, de l'espace confiné du bordel pour en faire une figure publique. Dans ce roman, le personnage de « la folle d'en face » travaille, elle a un emploi, elle circule dans la ville, elle évolue entre deux pôles socioéconomiques - l'extrême pauvreté, l'extrême richesse -, elle fait 
partie d'une communauté de quartier et sexuelle, elle joue un rôle politique aussi en aidant le Front Patriotique Manuel Rodriguez (FPMR) lors de l'attentat à Pinochet ${ }^{11}$. Dans ce roman, la folle entre dans la communauté politique, mais pas en qualité de « citoyenne exemplaire ». Ceci étant, la catégorie « littérature homosexuelle» n'est pas suffisante pour penser ce geste. Je prends ici mes distances avec le terme « homosexuel ", en tant qu'adjectif ou prédilection thématique. À mon sens, le geste de Lemebel est autre. C'est une opération d'écriture qui porte atteinte à la construction grammaticale, normée de la phrase. Il introduit une pulsion et une torsion à l'intérieur de la langue, en lui donnant un caractère sexué, en introduisant des excès onomatopéiques, des réitérations, des commutations, des restes d'oralité. Dans un texte récent, écrit en collaboration avec le chercheur argentin Fernando Davis ${ }^{12}$, nous avons appelé inflation baroque cette opération que l'on retrouve dans l'écriture de Lemebel et aussi dans celle de Perlongher ${ }^{13}$. Mais ce que je voudrais souligner, c'est que ce que remarque Raquel Olea au sujet du geste que réalise le roman de Lemebel dans la tradition narrative, on le retrouve dans les chroniques, mais aussi dans ses actions artistiques, à la fois dans son travail individuel et dans celui qu'il a réalisé avec Francisco Casas. Il faut rappeler que les «Yeguas del Apocalipsis » émergent en 1987, dans un contexte de dictature, marqué notamment par le pénalisation de la sodomie (en vigueur, au Chili, jusqu'en 1998) et l'irruption du VIH-sida, à une époque où les premiers mouvements homosexuels organiques n'avaient pas encore vu le jour. Il faudra attendre pour cela le

« Je veux un maquillage tout blanc même si je dois me refaire le portrait. Comme Ingrid Bergman dans Anastassia, comme Bette Davis dans Jezabel, comme une gamine, quoi, qui se serait endormie en attendant. J'aimerais que ce soit à l'aube, comme quand tu rentres du palais après avoir dansé toute la nuit. Surtout pas de messe, pas de curés, pas de blablas à vous faire bailler. Pas même un 'pardonnez-la, seigneur, cette pauvre tantouze a bien le droit d'entrer dans votre royaume'. Surtout pas de pleurs car je m'en vais bien payée, bien servie comme une chanteuse de boléro. Même pas besoin d'un requiem. Même pas besoin des baisers que l'amour n'a pas voulu me donner... Ni de l'amour. Regardez-moi ! Je traverse l'écume. Regardez-moi pour la dernière fois, bande d'envieuses! Car je ne reviens pas. $C^{\prime}$ est ça ma chance, je ne reviens pas. Je sens la soie humide de la mort me bander les yeux et je dis que j'ai été heureuse en cette dernière minute. D'ici, je n'emporte rien, parce que j'ai jamais rien eu, et même ça, je l'ai perdu ».

Extrait de : Lemebel P., «La noche de los visones (o la última fiesta de la Unidad Popular) », Loco afán. Selección de crónicas de sidario, Buenos Aires, Anagrama / Página 12, pp. 20-21.

11. Le FPMR fut le bras armé du PC chilien. Une de ses actions les plus célèbres a été l'attentat commis contre Pinochet en 1986, un épisode marquant des dernières années de la dictature (1973-1989).

12. Carvajal F. et F. Davis, «La inflación barroca. Contra-productivizaciones neobarrocas en Néstor Perlongher y Pedro Lemebel », in Los mil Pequeños Sexos, Buenos Aires, Ediciones UNTREF (sous presse).

13. Néstor Perlongher(1949-1992), poète, écrivain et militant lgbt argentin. 
début des années 1990. C’est pourquoi l'art, en tant que marque, ne parait pas non plus suffisant pour appréhender ses diverses productions. Le Manifeste et les premières actions des "Yeguas del Apocalipsis", telles que "La Conquête d'Amérique », qui propose une zone de douleur commune entre les victimes de la dictature et les victimes du sida, sont des faits de rupture, des évènements de rupture, qui ouvrent un espace d'intelligibilité et d'énonciation politisée pour la figure de « la folle »: une homosexualité masculine à la fois fragile et irritante, non «blanchie ». Et je crois que c'est là une inscription que Pedro Lemebel - son œuvre, mais aussi lui en tant que figure - laisse dans la culture et la société chiliennes, et qui déborde vers de multiples espaces de positionnement et d'intervention (que ce soit par rapport aux politiques de la mémoire, à la période de post-dictature, au néo-libéralisme, etc.). Par exemple, le lien d'amitié entre Pedro Lemebel et la dirigeante communiste Gladys Marín a été fondamental pour intervenir sur l'homophobie au sein de la gauche chilienne. C'est aussi le cas d'autres alliances avec des féministes ou des figures de la défense des droits de l'homme. Je l'ai connu dans les dernières années de sa vie, mais je crois que je peux dire que, dans le quotidien, chaque conversation avec Pedro te communiquait un peu de cette puissance. Il y avait chez lui quelque chose de très risqué, pas dans le sens du scandale, qu'il mâ̂trisait par ailleurs fort bien, mais plutôt dans le sens d'être capable de faire et de dire sans peur.

CEC : Concernant ce dernier point. Tu utilises le mot "intervenir ». Penses-tu que Lemebel est parvenu à modifier des perceptions, des comportements au sein de cette gauche ? En deçà ou au-delà de son amitié avec Gladys Marin, et d'autres personnalités très importantes, mon impression, à la distance, est que l'homophobie est restée aussi forte qu'autrefois mais maintenant au vu et au su de tout le monde. Lemebel ne l'a-t-il pas dévoilée ? Ne marquet-il pas une limite justement au sein de cette culture de gauche qui, dans d'autres aspects, se conçoit comme d'avant-garde ? S'il y a eu changement, où pourrait-il se situer?

F.C. : Je crois que l'opération que fait Pedro Lemebel par rapport à la gauche, dès son Manifeste, est complexe et peut avoir plusieurs lectures. Dans la langue révolutionnaire, le signifiant « homosexuel » a été lié historiquement à une série de figures : décadence bourgeoise, déviation morale, lâcheté, trahison. Dans ce langage, le fait d'être exclu de ce que les normes du genre ratifient comme masculin, équivaut à être banni du champ légitime de l'entraînement, des habiletés et des codes moraux propres à l'engagement militant. Ou, plus précisément, être banni de la scène de la passion politique, de l'engagement et de la possibilité d'assumer un risque au nom d'une cause. Autant d'expériences capturées par la figure du militant qui est passé sans dévier par tous les rites de la socialisation masculine. C'est pourquoi, dans le Manifeste de Lemebel, la virilité (hombría) - qui en espagnol désigne à la fois ce qui fait 
l'homme, le mâle, ce qui est proprement masculin, tout en étant synonyme de courage -, et la matrice sacrificielle qui conditionne l'appartenance à la gauche sont des nœuds qui ne marchent plus. Si on lit avec attention le Manifeste, on peut voir de quelle manière Lemebel désubstantialise la virilité, et la montre comme une puissante fiction, non comme une essence. La virilité apparait dès lors définitivement extérieure, comme quelque chose qui s'enseigne, qui s'apprend, quelque chose qu'on inculque, qui est reçu, quelque chose qui peut ne plus avoir cours. En opposition aux espaces hégémoniques de la virilité, tels que la caserne militaire ou le football, Lemebel revendique une virilité apprise " en esquivant les coups de couteaux dans les sous-sols du sexe par où je suis passé », c'est-à-dire, à l'intérieur des circuits secrets où transitent les pratiques abjectes du désir. Dans ce sens, il est même possible de penser que le fait de confronter l'homophobie c'est, pour Lemebel, un apprentissage du courage politique et, en même temps, une manière de subvertir la dichotomie courage = virilité $/$ lâcheté $=$ non virilité. Lorsqu'il écrit $:$ «Mon courage c'est de m'accepter différent / Être lâche c'est quelque chose de plus dur / Je ne tends pas l'autre joue / Je tends mon cul, camarade », Lemebel joue avec l'idée d'un courage sans virilité ou d'une virilité disloquée, il désactive ce qui soutient l'idéal masculin, une hiérarchie phallique. Ici Lemebel laisse en évidence le fait que le corps de l'homme est pénétrable et qu'il a le même statut que d'autres corps. C'est ainsi que Lemebel réclame, et en même temps démonte, de manière parodique l'homophobie et la matrice sacrificielle de la gauche. C'est un aspect de la chose.

"La Choumilou est morte le jour où la démocratie est arrivée, le pauvre cortège a croisé sur sa route la grande fête du triomphe du NON a en plein milieu de l'Alameda. Ça n'a pas été évident de traverser au milieu de cette foule de jeunes qui étaient là, les visages maquillés, avec leurs drapeaux aux couleurs de l'arc-en-ciel. Ils chantaient, ils criaient, euphoriques, ils embrassaient les folles qui s'en allaient aux funérailles de la Choumilou. Et pour un instant, deuil et allégresse, tristesse et carnaval se sont mêlés. Comme si la mort avait fait une halte sur le chemin et était descendue de son carrosse pour danser une dernière cueca $^{\mathrm{b}}$. Comme si on pouvait encore entendre la voix mourante de la Choumi, quand elle a su les résultats des élections : Vous direz bien des choses à la démocratie de ma part. Et on aurait dit que la démocratie en personne lui répondait, incarnée par les centaines de jeunes sans chemise qui sont montés sur le carrosse, ont sauté sur le toit, se sont accrochés aux fenêtres, ont sorti des bombes aérosols et ont peint tout le véhicule avec des graffitis qui disaient : Adieu Tyran ».

Extrait de : Lemebel P., "La noche de los visones... », op. cit., p. 21.

a Plébiscite d'octobre 1988 qui met un terme à la dictature. L'Alameda citée ci-après est l'avenue principale de Santiago.

b Danse nationale chilienne, danse de couple, particulièrement gaie (devenue aussi l'un des symboles de la dictature suite l'usage qu'en ont fait les familles -les femmes principalement- de "disparus " pour dénoncer l'absence de leurs proches). 
Je ne suis pas spécialiste de la politique interne du PC, mais je crois que l'amitié de Pedro Lemebel avec Gladys Marín (mais pas seulement) a contribué à révéler, à exposer l'homophobie, comme tu le signales, mais aussi à déplacer quelques frontières, à jeter une base politique en matière de politiques féministes et lgtb, sur lesquelles aujourd'hui il est très difficile de ne pas se prononcer, car il existe une communauté dans les rues devant laquelle il faut rendre des comptes. Un signe de cela est que le PC d'aujourd'hui (un PC qui, il faut le dire, est complètement aligné sur l'establishment politique), a une section consacrée au genre et à la diversité sexuelle. Le problème est qu'il ne s'agit pas seulement de la « représentativité » de certains secteurs ou causes, selon la grammaire partisane, mais aussi (de manière plus prégnante) de l'élargissement du champ du politique en intégrant des territoires comme celui de la sexualité, autrefois relégués en tant que pré-politiques. Cela n'implique pas une logique de l'addition, mais la possibilité d'ouvrir une brèche par où s'engouffre une force de transformation de l'ordre donné, dont le cours et les effets sont encore à voir. Dans ce sens, le fait que la gauche se prononce sur les droits sexuels et reproductifs n'est pas un point d'arrivée mais un point de départ qui, clairement, n'est pas suffisant, parce que les pratiques homophobes de violence et d'exclusion sont bien là (comme le montre la démission, au sein du PC, du dirigeant syndical homosexuel Cristián Cuevas en 2015), ainsi que dans toute la société. C'est une bataille permanente et ses enjeux se renouvellent quand l'homophobie coexiste avec des discours «politiquement corrects » ou quand les politiques lgtb se traduisent socialement comme un simple désir d'inclusion. Je crois que, s'il y a quelque chose que Lemebel permet de réactiver, c'est cette désobéissance à tout «blanchiment » de ce qu'on appelle « diversité sexuelle».

CEC : Je n'ai pas connu Pedro Lemebel personnellement. La manière dont j'ai découvert son cuvre est quelque peu insolite. J'ai vécu longtemps loin $d u$ Chili et, pour diverses raisons, je suis passée à travers, je jouais à cache-cache, pour ainsi dire, avec Lemebel. Puis, un jour, à Buenos Aires, au cours d'une conversation amicale, mon marchand de légumes s'écrie : "comment! Tu ne connais pas Lemebel, mais enfin, il faut absolument que tu lises Lemebel! ». Il m'a passé un livre. Ce marchand de légumes est quelqu'un de particulier, il chante, il fait du théâtre, il peint, c'est plus qu'un homme-orchestre, c'est un centre culturel à lui tout seul. Je n'ai pas en l'occasion d'en parler avec Pedro, mais je crois qu'il aurait aimé savoir que cet ami aimait ses textes. Le fait est que, dès que je l'ai lu, je me suis mise dans la tête de le traduire en français. Nous avons échangé quelques courriers. Nous avons pas mal discuté : fallait-il le traduire? Oui, non, pourquoi ? Je suis Chilienne, j'ai vécu longtemps en France. La sympathie de Lemebel envers les Chiliens de France était... très limitée... On ne pouvait rêver pire comme présentation... Bref, il a fallu argumenter. Je n'ignorais pas que la traduction de ses chroniques (vers n'importe quelle langue) est plus que difficile. Mais il me semblait qu'il fallait s'obstiner 
quand même. Comment penses-tu la singularité de son écriture? Non plus seulement dans les aspects que tu as signalés dans d'autres textes (voir plus loin dans le dossier "Arder "), pas dans leur dimension esthétique, comme si chaque geste d'écriture était aussi un type d'intervention, une marque, mais plutôt du point de vue de la possibilité d'un partage avec des lecteurs venus d'ailleurs? Je fais référence à cette question de la traduction.

F.C. : Je voudrais reprendre un fil de ta question. Il y a quelque chose de l'ordre de l'imprévu dans l'œuvre de Pedro : l'œuvre arrive, débarque, n'importe où. C'était quelqu'un qui aimait les marchés, les halles, il aurait aimé cette scène : cette introduction à son écriture de manière anonyme et quotidienne grâce à la parole persuasive d'un marchand de légumes, dans un quartier de Buenos Aires. Cette histoire rend bien compte de la capacité qu'avait Pedro, son œuvre, de produire des scènes improbables, d'habiter le sens commun de l'intérieur, de bouleverser des positions et des hiérarchies. Cette capacité à se situer non pas en dehors, ni au-dessus mais dans le sens commun, d'en

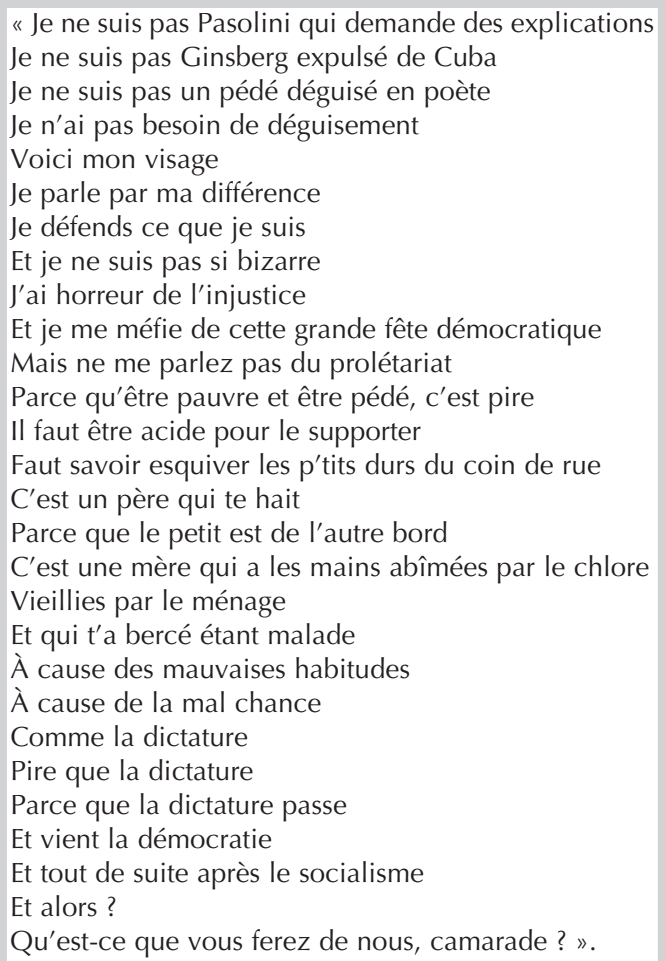

Extrait de : Lemebel P., "Manifiesto (Hablo por mi diferencia)", Loco afán, op.cit., p. 83. Le Manifeste est suivi de cette mention : "Ce texte a été lu comme intervention dans un meeting de la gauche en septembre 1986 à Santiago du Chili ». 
dévoiler l'étrangeté (mais aussi son caractère étranger), ce qui permet à son écriture d'entrer en contact avec des subjectivités bien divergentes. Il devient alors possible que toi et le marchand de légumes, avec vos différences de genre, de nationalité, de classe, trouviez là une zone de complicité. J'ai écouté il y a peu de temps, la réponse que Pedro donne à une auditrice dans l'émission de radio Triángulo Abierto en $1993{ }^{14}$. C'était une dame qui considérait humiliant que les homosexuels soient définis socialement seulement par leur sexe. Pedro répondait : "vous avez raison ». Mais tout de suite après, il soulignait la dimension homophobique de son affirmation. Il disait quelque chose comme : je ne veux pas être défini seulement par mon sexe, je veux pouvoir parler en me plaçant sur d'autres scènes politiques, mais l'homosexualité est un territoire politique d'énonciation qui doit être ouvert, occupé, défendu. C'était une manière de produire une horizontalité non complaisante.

Je crois que c'est une des dimensions de l'affaire, liée à l'un des aspects de son écriture, qui est faite de plusieurs textures et de plis. Et cela nous amène à la question de la traduction et ce que tu disais : la manière dont l'écriture de Pedro Lemebel opère une série de torsions, de zigzags et de frictions qui brisent ou fracturent l'usage normé de la langue pour ouvrir un mode d'énonciation marica ${ }^{15}$ (comme s'il perforait une langue pour en faire apparaitre une autre de l'intérieur), fait que son écriture est dans un certain sens marquée par le local ; et c'est de ce point de vue qu'elle prête peut-être une plus grande résistance à la traduction. Car, comme on dit, toute traduction implique trahison, une perte, un deuil. Au Chili, qui historiquement et géopolitiquement se situe parmi les sociétés réceptrices des théories et des littératures métropolitaines, nous le savons bien. Dans ce cas, il s'agit de faire savoir aux lecteurs des centres métropolitains qu'ils vont avoir affaire à cette opacité, à cette partialité, et à cette incomplétude, et qu'ils auront à se situer pour un moment en situation d'étrangeté, et de provincialisme, et à se livrer à un autre engagement et à un autre temps dans la lecture. Cela n'a pas empêché, bien entendu, une réception de la littérature de Lemebel, en particulier dans un contexte anglosaxon, où le travail de Fernando Blanco a eu un rôle stratégique ${ }^{16}$. La question à laquelle je ne suis pas sûre de pouvoir répondre, car c'est une tâche permanente, c'est comment garder vivant le caractère mordant de son écriture dans un contexte globalisé, qui tend à homogénéiser et à rendre équivalentes les productions culturelles, rapidement consommables, comme c'est arrivé, par exemple, avec les lectures qui assimilent un peu vite- et en effaçant d'autres généalogies locales - l'écriture de Lemebel au queer.

14. Triángulo Abierto a été définie par ceux qui l’ont conçue et réalisée-Victor Hugo Robles, Juan Pablo Suherland et Héctor Nuñez- comme la première émission de radio d'homosexuels et de lesbiennes au Chili. Diffusée par Radio Tierra où, des années plus tard, Pedro Lemebel a présenté sa propre émission : Cancionero.

15. Adjectif tiré de maricón : mot qui désigne un homosexuel dans le monde hispanophone. Désignation péjorative que Pedro Lemebel, avec d'autres, se réapproprie pour en faire autre chose qu'une insulte. 
CEC : Le numéro dans lequel cet entretien va paraitre est consacré à la nuit et la nuit est un élément omniprésent dans l'œuvre de Lemebel. Peux-tu nous en dire un mot?

F.C. : Dans une chronique sur le Premier Mai, Pedro Lemebel déclare son soutien au mouvement des travailleurs et dit qu'il aime ce jour car on ne travaille pas. Il affirme qu'il a toujours aimé les grèves, les pauses, les récréations, et qu'il n’a jamais partagé « ce plaisir quasi religieux du travail 17 ». « Je déteste le travail, je déteste les fourmis et les abeilles, bien soumises au patron, ces idiotes, ces esclaves ». La nuit de Lemebel est dans cette anti-économie. Dans la manière qu'a Lemebel de perforer l'ordre calculé de l'accumulation laborieuse de jour, avec une déviation et une perte, avec des formes d'enregistrer une précarité capable de gâchis et d'oisiveté, et bien entendu la sexualité marica, sa promiscuité et sa fugacité, la fête, le romantisme tragique, la cuite, le piège. Mais il y a aussi quelque chose de nocturne dans la mélancolie des chroniques de Lemebel, dans la manière qu'il a de se submerger dans ce qui reste des ombres des discours dominants, des récits historiques officiels, cette manière qu'a son écriture de rendre audibles des subjectivités, des plaisirs et des morts sur lesquels il y a eu un long silence social.

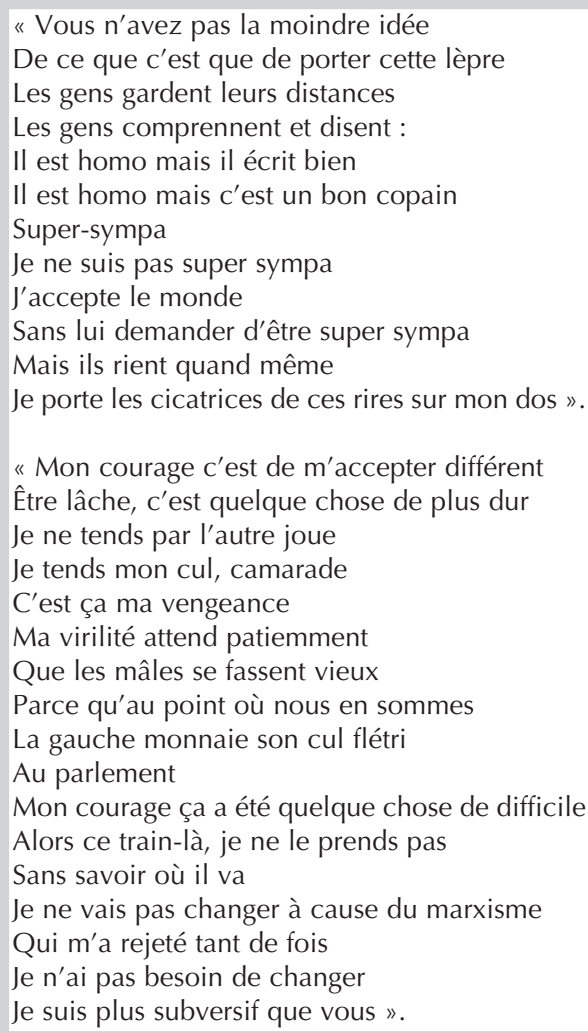

Extraits de : Lemebel P., " Manifiesto (Hablo por mi diferencia) ", Loco afán, op.cit., pp. 86-87.

16. On peut citer au moins deux ouvrages publiés par Fernando Blanco (le deuxième en collaboration avec Juan Poblete) qui peuvent être vus dans ce sens : Reinas de Otro cielo. Modernidad $y$ autoritarismo en la obra de Lemebel, Santiago, LOM, 2004 et Desdén al Infortunio. Comunicación y público en la narrativa de Pedro Lemebel, Santiago, Cuarto Propio, 2009.

17. Lemebel P., Háblame de amores, Barcelona, Seix Barral, 2013, p. 266. 